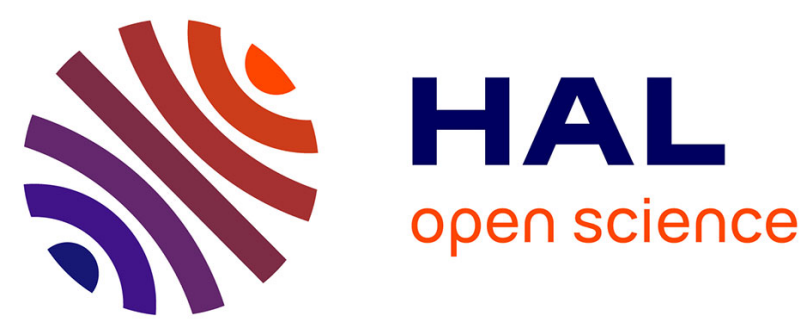

\title{
A Greedy Approach Combined with Graph Coloring for Non-Uniform Beam Layouts Under Antenna Constraints in Multibeam Satellite Systems
}

Jean-Thomas Camino, Laurent Houssin, Christian Artigues, Stéphane

Mourgues

\section{To cite this version:}

Jean-Thomas Camino, Laurent Houssin, Christian Artigues, Stéphane Mourgues. A Greedy Approach Combined with Graph Coloring for Non-Uniform Beam Layouts Under Antenna Constraints in Multibeam Satellite Systems. 2014 7th Advanced Satellite Multimedia Systems Conference and the 13th Signal Processing for Space Communications Workshop (ASMS/SPSC), Sep 2014, Livourne, Italy. pp.a55. hal-01068375

\section{HAL Id: hal-01068375 https://hal.science/hal-01068375}

Submitted on 29 Apr 2015

HAL is a multi-disciplinary open access archive for the deposit and dissemination of scientific research documents, whether they are published or not. The documents may come from teaching and research institutions in France or abroad, or from public or private research centers.
L'archive ouverte pluridisciplinaire HAL, est destinée au dépôt et à la diffusion de documents scientifiques de niveau recherche, publiés ou non, émanant des établissements d'enseignement et de recherche français ou étrangers, des laboratoires publics ou privés. 


\title{
A Greedy Approach Combined with Graph Coloring for Non-Uniform Beam Layouts Under Antenna Constraints in Multibeam Satellite Systems
}

\author{
Jean-Thomas Camino ${ }^{(1)(2)(3)}$, Stéphane Mourgues ${ }^{(1)}$, Christian $\operatorname{Artigues}^{(2)(3)}$, Laurent Houssin $^{(2)(4)}$ \\ ${ }^{(1)}$ Airbus Defence and Space, Space Systems, Telecommunication Systems Department, \\ 31 Rue des Cosmonautes, 31402 Toulouse, France \\ ${ }^{(2)}$ CNRS, LAAS, 7 Avenue du Colonel Roche, F-31400 Toulouse, France \\ (3) Univ de Toulouse, LAAS, F-31400 Toulouse, France \\ ${ }^{(4)}$ Univ de Toulouse, UPS, LAAS, F-31400 Toulouse, France
}

\begin{abstract}
Because of the ever-increasing traffic and quality demands for both internet and television, satellite systems must aim at designs that use the satellite resources in the most efficient way possible. In the case of multibeam satellite systems, this is achieved by making optimal use of the plurality of beams in terms of frequency reuse, power allocation, and quality of the layout. That last point is the one addressed in this paper, the optimisation of the beam layout being a complex but crucial task for the resulting telecommunication system since it directly affects its performances and cost. In the case of broadband systems, the key data is the repartition of the traffic demand over the zone to be covered which is never rigorously uniform. Though, it is common for satellite system design tools to rely on this fairly unrealistic assumption to provide regular coverage which is therefore often suboptimal : inappropriate beamwidths, overprovisioned or unsatisfied user stations, unprofitable beams. Nonetheless, one strong advantage of the regular scheme is that it is known to be compatible with the single feed per beam antenna constraint of minimum angular distance for all the couples of beams coming from the same reflector. The aim of this paper is to present a randomized multi-start heuristic to build a non-uniform layout, driven by the different telecommunication mission criteria and by the aforementioned antenna constraint that is dealt with by a graph recoloring procedure via local search and simulated annealing.
\end{abstract}

\section{INTRODUCTION}

The optimization of the design of multibeam satellite systems has received great attention in the past few years, the efficient exploitation of the resources becoming more and more determining to comply with a consistently growing need for better cost/bit ratios in satellite systems. Along with nonuniform frequency and power allocation that are not treated herein, one of the necessary steps for approaching the best possible profitability for the space segment is the optimization of the beam layout. Independently of these subsequent allocations, the performances and cost of a broadband satellite system are expected to differ notably from one beam layout to another according to how the heterogeneity of the traffic demand over the coverable region is handled. Indeed, an efficient algorithm should tend to a layout with an optimal number of beams and perfectly appropriate beamwidths and antenna gains (see Fig. 1 for a simple representation of the problem). This is the main flaw of the common technique that

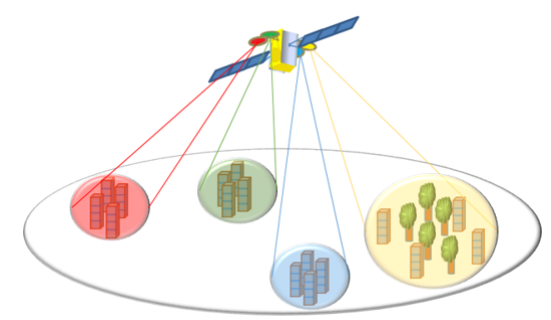

Fig. 1: Problem representation

uses a single beamwidth and the hexagonal lattice to cover the entire region (Fig. 2). That pattern is known to maximize the density of circle packing (see [3] for instance), but it would only be optimal for a traffic demand perfectly homogeneous in space and in intensity. On the other hand, technological feasibility has been proven for these layouts for a spacecraft equipped with 3 or 4 reflectors which is a strong enough reason to continue to rely on them regularly. Under the assumption adopted throughout this study that single feed per beam (SFB) antennas are used, the main technological constraint is that for each layout considered there must exist a mapping of the beams to the different reflector antennas for which the feeds do not overlap in the corresponding blocks of sources. In other words, an acceptable beam layout has corresponding source layouts that are feasible. This leads to a minimum angular distance from the satellite point of view between two beams associated to the same reflector antenna, that distance being a function of the size of the sources and therefore of the beamwidths at stake.

In the end, the real challenge here for any algorithmic procedure is to pertinently choose the beamwidths according to the density of the traffic demands, to place the beam centres with an antenna gain criterion while ensuring that the antenna constraint described above can be satisfied with an appropriate reflector to beam allocation. To that end, this paper aims at proposing a mathematical model for this constrained optimization problem, and a first algorithmic solution based on a randomized greedy approach to place the beams and graph coloring for the mapping between beams and reflectors. 


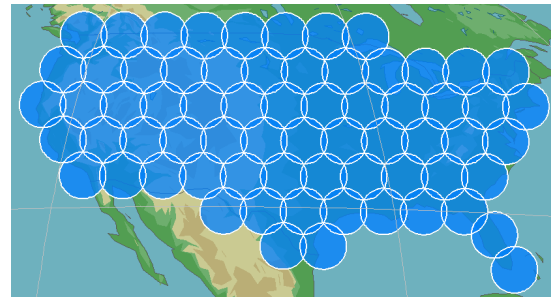

Fig. 2: Regular layout

\section{STATE-OF-THE-ART}

Concerning the interpretation of the traffic demand density to determine the beam diameters to use, the authors of [1] suggest to aim at a load-balanced distribution of these demands within the different beams through an adjustment of their widths, the main idea being that imbalances of traffic demands inside the beams can lead to user stations being in the end either overloaded or underloaded in terms of offered capacity. The method proposed uses a partitionning of the region into evenly loaded polygonal subparts, each one of these being then treated individually to determine what beamwidth should be used in it and where the corresponding beam should be placed through an analysis of its shape and surface, thus defining the full layout. In that work, the question of the mapping of the beams to the antenna reflectors is not addressed. Furthermore, in [2] the authors observed that a drawback of this last method is that several zones remain uncovered or poorly covered. To overcome this issue, artificial neural networks are used and more precisely self-organizing maps that treat all the traffic demands in order to define sub-areas, each sub-area corresponding to one of the available beamwidths. The reasoning behind this method is that using narrow beams on the densest zone is a way to increase the antenna gains and the frequency reuse where it is the most needed, while using larger beams in the least dense areas can be sufficient to reach compliance. Beams are then placed in each sub-area in such a way as to minimize the uncovered areas, the regular lattice being used locally. Once the beams are placed over the service zone, the authors tackle the issue of the antenna configuration with a reflector allocation to the beams that includes a maximization of the angular distances between the beams coming from the same antenna, that NP-hard problem being solved heuristically. But the major drawback of treating the placement of the beams and their allocation to the antenna reflectors sequentially is that one is very likely to discover that, with a layout built regardless of the antenna constraint, even the optimal mapping of the beams to the reflectors that maximizes the minimum angular distance between each couple of beams belonging to the same reflector can turn out to be technologically infeasible with SFB antennas. In that case, forcing such an implementation would require to use smaller sources than advised thus leading to a deterioration of the antenna performances through spill-over losses and important side lobes.

For the work presented here, particular attention has been paid to feasibility through a SFB technology and therefore to the satisfaction of these antenna constraints (see III for details) which are taken into account dynamically in the layout building procedure. Concerning the choice of the beamwidths, the idea of [2] of treating the input traffic demands is revisited but adapted to the mathematical model described further in the article. In practice, statistical discretization is operated on a set of data representing the density of traffic in the neighborhood of each possible centre for the beams to define in advance the beamwidths to use on these positions : narrow beams where the demand is dense, larger beams elsewhere. Guided by the combinatorial model of the problem, a heuristic procedure combining a randomized multi-start greedy approach and graph coloring is applied to find jointly interesting positions for the beams and an acceptable beam mapping to the reflectors with the additional constraint that the number of beams can be bounded.

\section{THE ANTENNA CONSTRAINT}

When designing a layout with single feed per beam antennas, the angular distance between two beam centres of two beams generated by the same reflector cannot be lower than a value determined by the size of the two corresponding feeds. Indeed, having close beam centres implies having close corresponding sources in the block of sources of the reflector in question. When two sources of two given sizes become tangent, the minimum possible distance between the two horn centres is reached, situation that leads through the reflection by the parabola to the minimum angular distance between the two emitted beams. This optical property is known under the name "beam deviation" and has led to the definition of the "beam deviation factor", denoted here by $\Gamma$. The simple offset reflector system presented in Fig. 3 illustrates the phenomenon. Here is some notation used in the equations that follow :

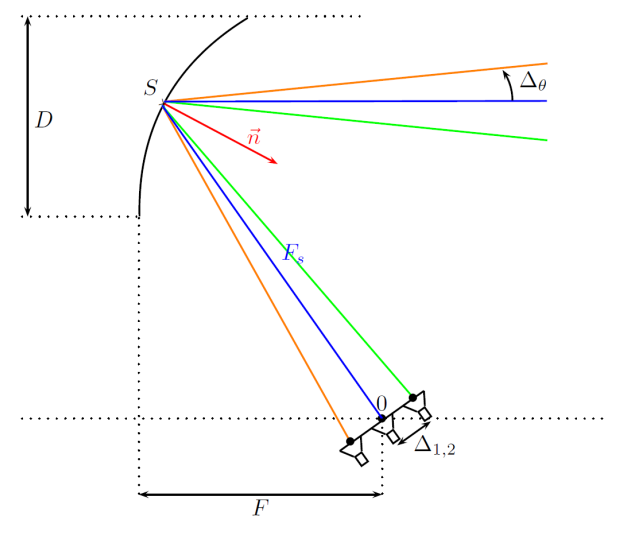

Fig. 3: Simple offset reflector system and beam deviation

$\diamond \quad \delta$ : source diameter

$\diamond \quad \Delta_{i, j}:$ distance between the two horn centres labelled $i$ and $j$

$\diamond \quad \Delta_{\theta}:$ angular distance between the beams

$\diamond \quad F$ : focal length

$\diamond \quad F_{s}:$ slant focal length

$\diamond \quad D$ : diameter (projection of the parabola diameter on the focal plane)

$\diamond \quad$ Point $O$ : focus 
From [6], for two horns $i$ and $j$, we learn that there exists $\alpha<1$ such as :

$$
\Gamma=\frac{\arcsin \left(\frac{\Delta_{i, j}}{F_{s}}\right)}{\arctan \left(\frac{\Delta_{i, j}}{F_{s}}\right)} \frac{1+\alpha\left(\frac{D}{4 F_{s}}\right)^{2}}{1+\left(\frac{D}{4 F_{s}}\right)^{2}}=\frac{\Delta_{\theta}}{\arctan \left(\frac{\Delta_{i, j}}{F_{s}}\right)}
$$

Furthermore, since $\frac{F_{s}}{D}>1,5$ in satellite architectures, the approximation $\Gamma \approx 1$ is known to be legitimate which leads to the following formula for the angular deviation :

$$
\Delta_{\theta}=\arctan \left(\frac{\Delta_{i, j}}{F_{s}}\right)
$$

The next step would be to derive $\Delta_{i, j}$ according to the widths of the beams $i$ and $j$, which is not done here. On that regard, the important property is that using larger beamwidths requires larger sources to concentrate the illumination on a reduced reflector surface, which leads to greater minimum inter-sources distances. Finally, note that since the problem is solved with a finite number of possible beamwidths fixed in advance, all the minimum angular separation values can be computed in preprocessing.

\section{THE PROBLEM MODEL USED}

\section{A. Input data}

As already discussed, in broadband satellites the telecommunication mission is defined by the traffic requests over the geographic area treated. The corresponding users are aggregated in what will be called stations to be in the end represented by a reasonably large number of points at the surface of the Earth. Each station can be identified for instance with its true view angles $\left(\theta_{x}, \theta_{y}\right) \in \boldsymbol{\Theta}_{\mathbf{x}} \times \boldsymbol{\Theta}_{\mathbf{y}}$. One can therefore define a discrete and finite set $\mathcal{S} \subset \Theta_{\mathrm{x}} \times \Theta_{\mathrm{y}}$ that contains all these station coordinates. We can then define a function $T$ as follows :

$$
\begin{aligned}
T: \mathcal{S} & \rightarrow \mathbb{R}^{+} \\
s & \mapsto T(s)=\text { Traffic of the station at } \mathrm{s}
\end{aligned}
$$

\section{B. Set of the possible positions for the beams}

The choice of the set of potential beam centres is of high importance since it determines the type of optimization procedures that will be usable. The main choice is whether to use a discrete or a continuous set. As discussed in the last paragraph, the nature of the traffic demands which are represented by a finite number of stations on the Earth's surface is the source of two determining properties for any function that returns a value linked to the overall traffic demand inside the coverable region : it is non-differentiable in most cases and always non-convex. The discontinuity property is what makes it impossible to rely on all the non-linear optimization techniques and the convexity would have been needed to use convex optimization methods that can deal with such non-differentiable objective functions. Smoothing the objective function with an accurate model of antenna gains could solve the non-differentiability, but then one would have to treat a highly multi-dimensionnal continuous problem. In the end, the choice of the discretization of the beam centres set was probably the most appropriate one. To do so, the idea is to analyze the $\mathcal{S}$ set of all the $\left(\theta_{x}, \theta_{y}\right)$ station coordinates in order to define a meshing area. To do so, a margin is taken on the boundary of that set and a uniform grid is generated with two tunable space steps in the $\theta_{x}$ and $\theta_{y}$ directions. Let $\mathcal{C} \subset \boldsymbol{\Theta}_{\mathbf{x}} \times \boldsymbol{\Theta}_{\mathbf{y}}$ be the discrete and finite resulting set of potential centres for the beams that will be used throughout this study.

\section{Combinatorial model}

Because of this discretization of the possible positions for the beams (corresponding set denoted by $\mathcal{C}$ ), along with the finite number of reflectors and beamwidths (corresponding sets $\mathcal{R}$ and $\mathcal{W}$ respectively), the problem belongs to the class of combinatorial optimization problems. A modelling phase lead to a representation where each possible position for the beam centres is seen as a vertex of a graph in which there is an edge between each couple of vertices considered to be too close to be the two centres of two beams assigned to the same antenna reflector. On that matter, note that the beamwidths to use in each centre and therefore the minimum angular distances to be satisfied are preprocessed (see V) for all the couple of beams. Let $k$ be the number of available reflector antennas on the satellite, each one of them can be seen as a different "color" in the graph terminology. Then, extracting from the graph of the possible positions for the beams a subgraph legally colored with $k$ colors (assignment of colors to the vertices in such a way that no adjacent vertices use the same color) is equivalent to finding an acceptable layout with respect to the antenna constraints. Note that the cardinality of this subgraph is also limited by the number maximum of beams than can be used. Then, one has to define an objective function that can be computed for each acceptable set of beam positions to define the optimization problem as follows : find the $k$-colorable subgraph that is optimal according to the telecommunication criteria. On that regard, many different approaches can be considered, ours being detailed in IV-D. Whatever the objective function, Fig. 4 provides a visual representation of the decision variables, of the constraints, and of the criteria. There is not,

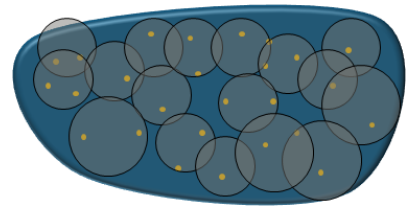

(a)

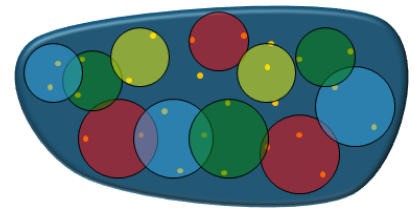

(c)

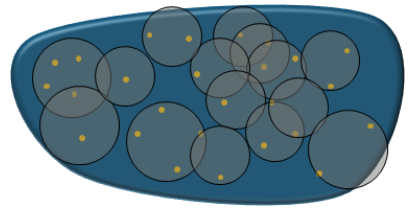

(b)

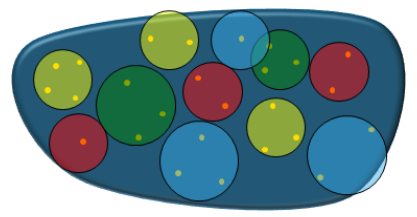

(d)
Fig. 4: (a) Too many beams (b) Impossible assignment to $k$ reflector antennas (c) Non-optimal acceptable solution (d) Optimal solution

to our knowledge, literature that directly addresses a problem strictly similar to the one described in this article. The closest we could find were the $k$-centre problem [5] and the max $k$-cut problem [4]. The former tries to find locations among a finite 
number of possibilities for a limited number of warehouses with an objective linked to the distances to the cities they have to serve. The warehouses can be seen as the equivalent of our beams, but their positions do not have to satisfy any kind of separation constraint comparable to the antenna constraint. Concerning the $k$-cut problem, the goal is to partition the set of vertices of a given graph in such a way that a maximum number of edges are cut i.e. with corresponding vertices that belong to two different subsets of the partition. The main flaw of this approach for our problem is that the resulting coloring is not legal i.e. adjacent vertices might use the same color and therefore one would have to treat each output to reach an acceptable set of beams according to the antenna constraint, so that option is not entirely satisfactory. In the end, it has been chosen to turn to an ad hoc heuristic described and analyzed in the remainder of the article.

\section{Objective function considered}

The role of the objective function is to translate the needs into a real-valued function whose minimization (it could have been a maximization) brings us closer to their satisfaction. The variables of that function are all the system parameters that have to be optimally determined. More precisely, with

$\diamond \quad n_{\max } \in \mathbb{N}$ the maximum number of usable beams

$\diamond \quad \forall i \in \llbracket 1, n_{\max } \rrbracket, c_{i} \in \mathcal{C}$ the position of the centre of the $i^{t h}$ beam

$\diamond \quad \forall i \in \llbracket 1, n_{\max } \rrbracket, w_{i} \in \mathcal{W}$ the beamwidth of the $i^{t h}$ beam

$\diamond \quad \forall i \in \llbracket 1, n_{\max } \rrbracket, r_{i} \in \mathcal{R}$ the index of the reflector from which is generated the $i^{\text {th }}$ beam (only involved in the constraints, not the cost function)

$\diamond \quad \forall i \in \llbracket 1, n_{\max } \rrbracket, b_{i} \in \mathcal{B}=\{0,1\}$ the boolean variable that indicates whether the $i^{\text {th }}$ beam is actually used or not

we can define the global variable of our problem as follows:

$$
X=\left(\begin{array}{c}
c_{1} \\
w_{1} \\
r_{1} \\
b_{1} \\
c_{2} \\
\vdots \\
c_{n_{\max }} \\
w_{n_{\max }} \\
r_{n_{\max }} \\
b_{n_{\max }}
\end{array}\right) \in(\mathcal{C} \times \mathcal{W} \times \mathcal{R} \times \mathcal{B})^{n_{\max }}
$$

As it has been already said before, choices on the criteria to consider had to be made to address the problem. Here is the list of the properties sought for the minimized functional, followed by details on their relevance :

1) A functional that decreases when the traffic demands inside the beams increase.
2) A functional that is additive with respect to the different beams, i.e. that computes a certain value for each beam (linked to the traffic demand inside the beam) and sums them all up.

3) A functional in which the impact of the traffic of a given station in a beam decreases with the distance of that station to the beam centre.

4) A functional in which stations that are covered by more than one beam at the same time only contribute to the overall traffic demand (and thus to the corresponding functional decrement) of one of these beams.

The first property is the most obvious one since it is what will directly lead to a maximization of the traffic coverable by the beam layout. An additive functional leaves the door open to easy implementations of greedy type algorithms on which interesting literature has been found (see [7] and [8]). The reduced impact of the stations far from their beam centre is a way to take into consideration the decrease of capacity resulting from the loss of antenna gain. The last criterion used is probably the most questionable one : it presupposes that the traffic demand of a station cannot be dealt with by more than one beam. Therefore, potentially interesting overlapping situations are not handled in that first approach and some station requests might remain partially unsatisfied for that reason. But this property for the functional is necessary to avoid an agglomeration of all the beams in the densest area with a greedy approach. Therefore, for a station covered by more than one beam, the choice here has been to affect the corresponding traffic to the lowest-indexed of these beams (which is adapted to any heuristic that treats the variables in the order defined by the index $i \in \llbracket 1, n_{\max } \rrbracket$, the greedy type algorithms being good compatible examples once again). To explicitly include this choice in the functional expression, let us denote for all $i \in \llbracket 1, n_{\max } \rrbracket$ :

$$
\mathcal{S}_{i}=\left\{s \in \mathcal{S} \mid\left\|s-c_{i}\right\|_{2}<\frac{w_{i}}{2} \wedge \forall j<i,\left\|s-c_{j}\right\|_{2} \geq \frac{w_{j}}{2}\right\}
$$

Therefore, to be in $\mathcal{S}_{i}$, a station must be in the zone covered by the $i^{t h}$ beam, but not in any of the zones covered by the beams whose indices are less than $i$. Since it has been chosen to minimize a functional, one last notation is needed. Thus, let

$$
\mathbf{T}=\sum_{s \in \mathcal{S}} T(s)
$$

be the total traffic request over the studied area. Finally, let

$$
\mathcal{F}:(\mathcal{C} \times \mathcal{W} \times \mathcal{R} \times \mathcal{B})^{n_{\max }} \longrightarrow \mathbb{R}^{+}
$$

be the minimized objective function, defined as follows for all $X \in(\mathcal{C} \times \mathcal{W} \times \mathcal{R} \times \mathcal{B})^{n_{\max }}:$

$$
\mathcal{F}(X)=\frac{1}{\mathbf{T}} \sum_{i=1}^{n_{\max }}\left(\mathbf{T}-b_{i} \sum_{s \in \mathcal{S}_{i}} T(s)\left(1-2 \frac{\left\|s-c_{i}\right\|}{w_{i}}\right)\right)
$$

In $\mathcal{F}$, the traffic coverable by each beam is computed, with a first linear model of attenuation of the contribution of the 
stations far from the center. The global traffic $\mathbf{T}$ is used for two reasons : to transform the maximization of the traffic into a minimization problem, and to make sure that only positive values are computed and summed up. One can notice that the more active beams (i.e. $b_{i}=1$ for many $i$ ), the lower the functional, but the constraints will be limiting on that regard.

\section{PREPROCESSING FOR THE BEAMWIDTHS}

Let us remind that the width of the beams is an important parameter to determine to use efficiently the satellite resources. Indeed, using narrow beams in high traffic demand zones results in interesting antenna gains and frequency reuse schemes that are needed to comply with the capacity requests. Larger beams are expected to offer satisfying traffic in less dense zones. Therefore, the challenge is to be able to pertinently fit the repartition of traffic demand over the treated zone. The goal is to divide the entire region into subareas, each one corresponding to a possible beamwidths, that information being used afterwards when the beams are placed. Thus, we are following the idea of the authors of [2] but with the difference that the data sorting is applied to all the possible positions for the beam centres that are weighted according to the demand density in their vicinity, and not on the demanding user stations themselves. Furthermore, instead of turning to neural network, simple statistical discretization has been selected for its ease of implementation and its satisfying results. This technique that is often used by statisticians to create population density maps consists in partitioning the set of encountered density values into as many intervals as there are categories available for the data sorting, each subset of values being associated to one of these. In our case for instance, the lowest values of density will be associated to the largest beams. Concerning the size of the intervals, two techniques were examined :

- regular discretization : same size for all the intervals of the partition.

- $\quad$ arithmetic discretization : these sizes follow an arithmetic progression.

Given that the highest traffic demands are often concentrated in small areas, we often have to deal with a distribution of density values that is skewed to the right, so the arithmetic approach is a better fit in that case to reach a more or less balanced repartition of the different beam diameters over the zone. Yet, to make this directly usable, we had to compute a value representative of the density in the vicinity of every possible position for the beam centres. That is the role of the density function $\mathcal{D}: \mathcal{C} \rightarrow \mathbb{R}^{+}$defined as follows for all the possible centres $c \in \mathcal{C}$, where $\omega_{\max }$ is the largest beamwidth envisaged and $D_{c}=\left\{s \in \mathcal{S} \mid\|s-c\|<\frac{1}{2} \omega_{\max }\right\}$ is the set of user stations in a beam of diameter $\omega_{\max }$ placed in $c$ :

$$
\mathcal{D}(c)=\frac{4}{\pi \omega_{\max }^{2}} \sum_{s \in D_{c}} T(s)\left(1-2 \frac{\|s-c\|}{\omega_{\max }}\right)
$$

In the end, $\mathcal{D}$ has the following interesting properties :

- when the traffic demand is high in the vicinity of a given centre, $\mathcal{D}$ is also high. These high values can be due to a multiplicity of stations of low demands or to a few high demanding stations.
- when a demanding station is far from the centre, its influence on the density computed is smaller. This property was needed to choose adapted diameters, since without it, one could have to place narrow beams on centres considered as dense only because of stations on the periphery of the $\frac{1}{2} \omega_{\max }$ radius disk, none of which being actually coverable by such small beams.

The results presented all along this article have been obtained on a realistic but fictitious broadband scenario over Africa. In Fig. 5, this statistical discretization has been applied on Africa and Eastern Africa with respectively 2 and 4 available beamwidths.

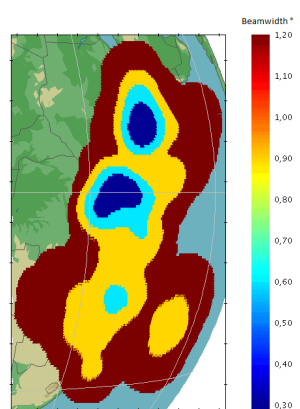

(a)

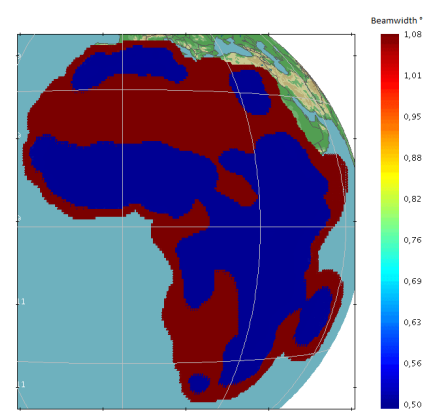

(b)
Fig. 5: (a) 4 diameters over Eastern Africa (b) 2 diameters over Africa

\section{THE ALGORITHMIC SOLUTION PROPOSED}

Let us remind that building algorithmically a layout implies solving jointly the following subproblems :

- The choice in advance of the beamwidths to use on each potential position for the beams already detailed in the last section.

- The progressive positioning of the beams on the possible centres.

- The allocation of a reflector to each beam, without violating the constraints.

In the approach proposed, they are tackled more or less independently with a multi-start greedy algorithm to position the beams, statistical discretization to choose appropriate diameters, and a graph coloring algorithm for the reflector allocation.

\section{A. Randomized multi-start greedy algorithm}

One big challenge of the beam layout problem is to define procedures that allow to efficiently position numerous beams from scratch. Different strategies can come to mind :

- defining the layout beam by beam.

- defining the whole layout with predefined patterns and blocks of beams.

The second option is exactly what is done today with the regular patterns whose suboptimality has already been discussed. The strategy here will therefore be to try to define 
a layout beam by beam. An exhaustive tree search was clearly not an option given the size of the search space after a sufficiently refined meshing of the zone, a greedy approach being preferred to create acceptable layouts in a reasonable amount of time. However, constraint programming and more precisely constraint propagation were inspirational for the definition of our constructive heuristic. Indeed, to deal with the minimum angular distance constraint, our choice has been to propagate it through an update of the non-usable values for the reflector variable each time a beam is positioned. That way, we cannot use any value that would lead to a violation of the constraints. In practice, to apply the greedy approach (optimal local choice without questioning the previous ones, see [7]), the increment of the new beam for $\mathcal{F}$ is computed for all the possible centres, which is immediate since the functional is additive, and a list of the better possibilities is drawn up. Instead of using a standard greedy approach, randomization is operated like in [8] through a random choice of the next beam positioned in a list of the best potential beams. With a "multistart"-like approach, each run produces rapidly a new solution that is different than the previous one, these solutions thus exploring zones of the search space that the standard greedy approach could not have seen, the goal being to do better than the latter. An overall view of the algorithm steps could be :

- Determination of the beamwidths to use in each possible beam centre

- Random choice of the position of the first beam in a list of best possibilities

- Reflector assignment to the first beam

- Random choice of the next beam, reflector assignment to that beam

- And so on until it is not possible to place another beam (impossible reflector assignment even with recoloring, or no interesting positions left)

In the end, the algorithm used is the one described in pseudocode in Fig. 6.

\section{B. First-fit like graph coloring with simulated annealing for reflector allocation}

The strength of the algorithm proposed is to take into account the antenna constraint dynamically which is a way to ensure that the layouts obtained are feasible. To build such an algorithm, it has been decided to define for each possible centre $c \in \mathcal{C}$ a matrix of ones and zeroes $M^{c} \in$ $\mathcal{M}_{\text {card }(\mathcal{R}), \operatorname{card}(\mathcal{W})}(\{0,1\})$ in which the impact of the already positioned beams in terms of usable reflectors for the potential beam centre $c$ can be stored and read. All these matrices are updated each time a beam is added. Thus, at any moment during the algorithm, for a given centre $c$ and for the $j^{\text {th }}$ beamwidth, $M_{i, j}^{c}$ indicates whether the $i^{t h}$ reflector can be used to place a new beam without violating a minimum angular distance constraint. When positioning a new beam at $c$, the subgraph described in IV-C evolves, and when at least a reflector is naturally available for this new node according to the $M^{c}$ matrix, the less used by the already placed beams is picked (random choice when there are ties). At that point, we had to decide what to do when facing a situation where nb_of_greedy_layouts $=0$

while nb_of_greedy_layouts $<$ nb_of_greedy_layouts_wanted do

nb_of_beams_placed $=0$

while nb_of_beams_placed $<n_{\max }$ do

if $\exists$ beam positions with non-zero traffic demand then

Greedy establishment of the list of best candidates

Random choice among this list

Allocation of reflector (potential recoloring)

if Beam not rejected then

nb_of_beams_placed++

traffic_covered+=traffic_covered_by_new_beam

end if

else

Break while loop

end if

end while

nb_of_greedy_layouts++

end while

Fig. 6: Multi-start randomized greedy layout generation

none of the satellite reflectors is usable. The natural reflex would be to forbid such centres and try those where there are available reflectors. However, it is important to notice that there is absolutely no reason that the way the graph has been colored until that moment should be optimal (in terms of minimum number of colors used), and even if it was, the newly added node and the corresponding edges have every chance to modify completely the structure of the optimal coloring. Therefore, trying to recolor the already existing beams may be a way to find a correct reflector allocation that includes the new beam. To do so, one has to decide which nodes have to be discolored. The choice here has been to define a tunable complexity $h \in \mathbb{N}^{+*}$ for the heuristic :

- For $h=1$, the direct neighbors of the problematic node are discolored.

- For $h=2$, the direct neighbors of the problematic node, as well as the direct neighbors of each one of them are discolored.

- $\quad$ etc...

This is illustrated in Fig. 7 where it is assumed that four reflector antennas are available.

As far as what concerns the graph recoloring algorithms themselves, each time a problematic node is found, it has been decided to use the first-fit algorithm and the simulated annealing algorithm on the subgraph constituted of all the discolored nodes and their direct neighbors (some of them being possibly already colored). The principle of the first-fit algorithm is to follow an ordered list of nodes that have to be colored, treat them one by one by assigning the first color available among an unlimited number of ordered colors. The order in which the nodes are colored is determining for the number of colors that will ultimately be needed (see Fig. 8 with the order red $<$ blue $<$ yellow $<$ green). The simulated annealing algorithm is only used with a local search on the order of the nodes when the first-fit algorithm did not provide a satisfying coloring of the graph. 


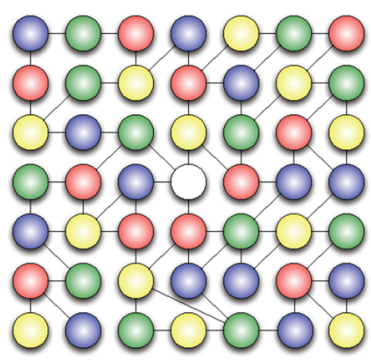

(a)

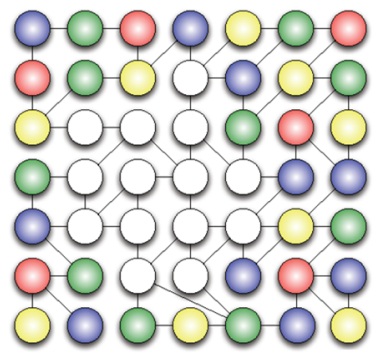

(c)

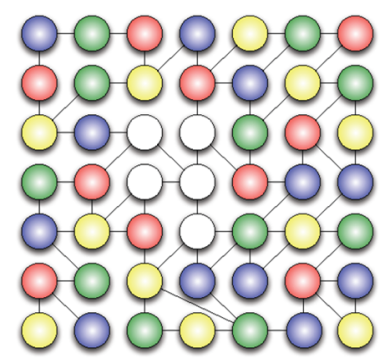

(b)

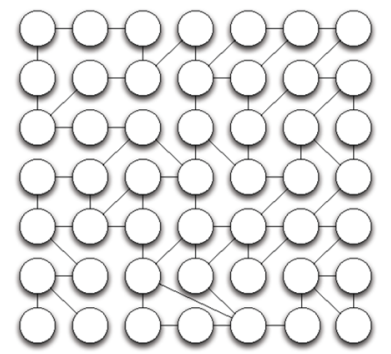

(d)
Fig. 7: (a) No reflector available (b) $h=1$ (c) $h=2$ (d) $h \rightarrow+\infty$

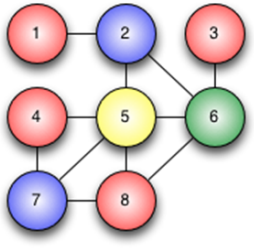

(a)

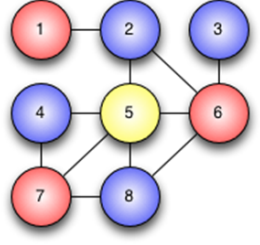

(b)
Fig. 8: (a) First-fit applied to the order $[1,2,3,4,5,6,7,8], 4$ colors (b) First-fit applied to the order $[7,6,2,5,4,8,3,1], 3$ colors

\section{RESULTS}

A. The antenna constraint and its corresponding graph coloring problem

As it was already said, a first-fit algorithm was used for the recoloring and when it was not enough to free space in the reflectors source blocks it was combined with a simulated annealing procedure. The first thing to do was to calibrate empirically the heuristic complexity $h$ presented above in order to optimally use the program during the campaign of tests on typical scenarios that followed. On figure 9, the evolution of the mean number of situations resolved by the graph coloring problem with the complexity $h$ is visible. The conclusion is immediate : $h=3$ should be used. It seems indeed to be a good tradeoff between situations where too few beams are discolored leaving not many options to try to find a valid recoloring and situations where too many nodes are discolored making the recoloring problem bigger and therefore harder to solve, at least with our first-fit like algorithm. A campaign of 1000 runs on a broadband scenario over Africa where 150 beams could be placed at most allowed to obtain the following results for the recoloring procedure with a complexity $h=3:$ in average,

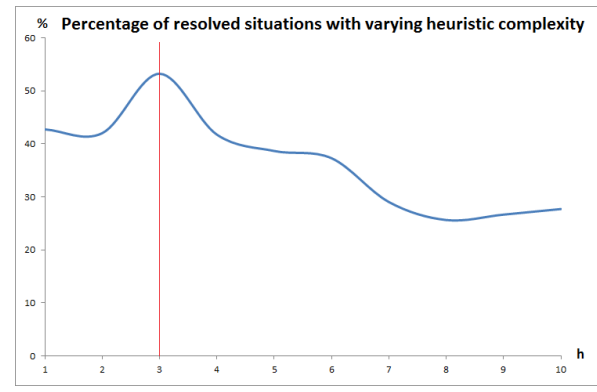

Fig. 9: Mean percentages of resolved situations with the graph coloring procedure for different $h$ values

$42 \%$ situations where no reflectors were available were solved by the first-fit recoloring, and $44 \%$ of those it did not solve were then solved by the simulated annealing recoloring. In the end, the results are encouraging and strengthen the idea that finding good recoloring algorithms can lead to layouts where interesting beams are less rejected.

\section{B. Results on the randomized multi-start approach}

The multi-start approach was the one chosen to try to improve the performances of the standard greedy algorithm. To measure the added value of that algorithm, we asked for 200 different greedy solutions on the same scenario and we compared all the resulting objective function values to the one we get after applying a standard greedy algorithm. The results are shown in figure 10 . We observe that only 3 greedy so-

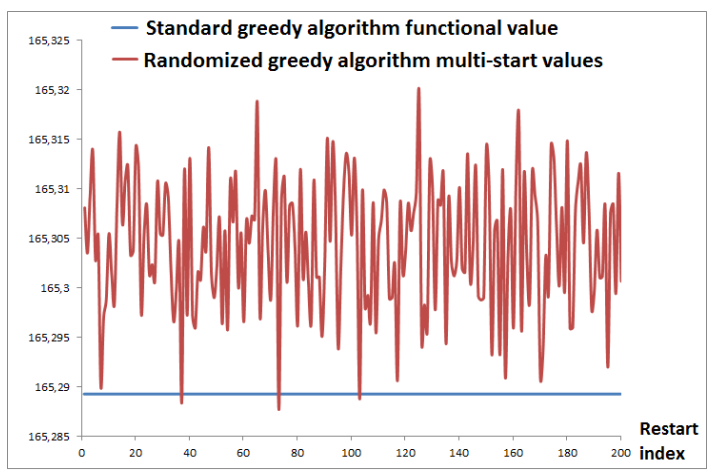

Fig. 10: Multi-start results compared to the standard greedy algorithm

lutions produced by the multi-start randomized approach beat the performances of the standard greedy algorithm, and the difference isn't really pronounced. At that point of the study, it is premature to conclude whether this slight improvement is significant for the telecommunication system, complete system designs using the greedy layout and its improved versions needing be compared.

\section{Global results on typical scenarios}

Several scenarios were tested : North Africa, Austral Africa, Eastern Africa and Africa (whole continent). The corresponding solutions returned by the algorithm can be seen on the figures that follow. The North and Eastern Africa 


\begin{tabular}{|c|c|c|}
\hline Angular beamwidth & $\mathbf{0 , 5 ^ { \circ }}$ & $\mathbf{1}, \mathbf{0 8}^{\circ}$ \\
\hline $\mathbf{0}, \mathbf{5}^{\circ}$ & $0,82^{\circ}$ & $1,28^{\circ}$ \\
\hline $\mathbf{1}, \mathbf{0 8}^{\circ}$ & & $1,76^{\circ}$ \\
\hline
\end{tabular}

TABLE I: Angular constraints for the tested scenarios

scenario have been treated with only one possible beamwidth. For Austral Africa and the entire continent, two beamwidths were used. More precisely, we had :

- inhomogeneous traffic demand over the region (the colored background map in the figures)

- 4 reflector antennas available

- $\quad$ the maximum number of beams equal to 175

- $0,5^{\circ}$ and $1,08^{\circ}$ as available beamwidths

- $\quad$ the minimum angular distances of Table I

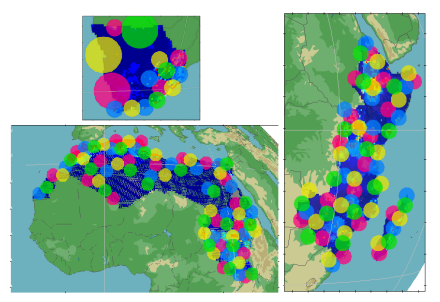

Fig. 11: Beam layout with one beamwidth generated over North-Africa

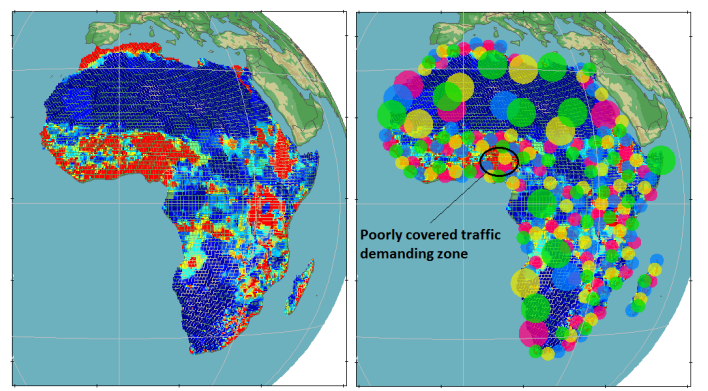

Fig. 12: Beam layout with two beamwidths generated over Africa

In all the scenarios presented, the coloring constraint (combined with the obvious constraint to not position beams that do not cover any station) is the one that ends the generation procedure. As a consequence, we can observe that some dense zones remain uncovered (see Fig. 12), and a finer analysis showed that the algorithm tried to place beams but failed because of the antenna constraints. Once again, the conclusion is that trying to implement better recoloring algorithm would have a direct impact on the quality of the layout. Fig. 13 provides the layout returned by the program when the constraint isn't taken into account : one can notice that the greedy algorithm is indeed attracted by these zones but with the minimum angular distance constraint, most of these beams have to be rejected.

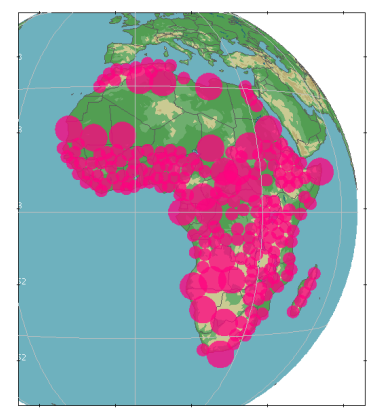

Fig. 13: Beam layout over Africa returned by the unconstrained algorithm

\section{CONCLUSION}

In the end, this paper provides an alternate option for building non-uniform layouts with a dynamic consideration of the single feed per beam antennas constraints, the good RF performances of this technology making it an interesting solution for the multibeam systems. What has been presented here is the first step of a work that will include frequency and power allocation to the beams, link budget assessments and capacity computations to finalize this system optimization but even without that level of accuracy regarding the system performances, this work provides a tool to build irregular layouts algorithmically with a guarantee of feasibility for an implementation with SFB antennas. Also, the difficulties of satisfying predefined angular separation constraints for the beams of the same antenna while simultaneously trying to ensure the contiguity of coverage have been highlighted. At that point, layouts that use beam diameters adapted to the density of the zones covered and that optimize the antenna gains for the user stations can be reached but they might have the main flaw of leaving some economically important regions or cities uncovered. Though, improvements on the graph coloring procedure might solve some of these unacceptable situations making this approach more efficient.

\section{REFERENCES}

[1] P. Angeletti and J. Lizarraga, Non-regular Multibeam Coverage Payloads for Non-Uniform Traffic Demand. $2^{\text {nd }}$ ESA Workshop on Advanced Flexible Telecom Payloads. Noordwijk, Netherlands, April 2012

[2] A. Kyrgiazos, B. Evans, and P. Thompson, Irregular beam sizes and non-uniform bandwidth allocation in HTS multibeam satellite systems. $31^{\text {st }}$ AIAA International Communications Satellite Systems Conference (ICSSC). Florence, Italy, October 2013

[3] H. Chang, A Simple Proof of Thue's Theorem on Circle Packing. September 2010

[4] A. Coja-Oghlan, C. Moore and V. Sanwalani, MAX k-cut and Approximating the Chromatic Number of Random Graphs. Springer, Lecture Notes in Computer Science, volume 2719. 2003

[5] R. Brandenberg and L. Roth, New Algorithms for k-center and Extensions. Springer, Lecture Notes in Computer Science, volume 5165. 2008

[6] Y. T. LO, On The Beam Deviation Factor of a Parabolic Reflector. IRE Transactions on Antennas and Propagation. February 1960

[7] D. Keil, Greedy and Other Efficient Optimization Algorithms. March 2005

[8] R. Marti, Multi-start Methods for Combinatorial Optimization. AT\&T Labs Research Technical Report. March 2012

[9] P. Borowiecki, Dynamic Coloring of Graphs. Journal Fundamenta Informaticae. April 2012 